\title{
Nematicidal Activity of the Endophyte Serratia ureilytica against Nacobbus aberrans in Chili Plants (Capsicum annuum L.) and Identification of Genes Related to Biological Control
}

\author{
Arnoldo Wong-Villarreal ${ }^{1, *}$, Erick Williams Méndez-Santiago ${ }^{2}$, Olga Gómez-Rodríguez ${ }^{3, *} \mathbb{C}$, \\ Liliana Aguilar-Marcelino ${ }^{4}$ (D) Daniel Cerqueda García ${ }^{5}$, José Q. García-Maldonado ${ }^{6}$, \\ Victor M. Hernández-Velázquez ${ }^{2}$, Gustavo Yañez-Ocampo ${ }^{7}$, Saúl Espinosa-Zaragoza ${ }^{8}$ (D), \\ Sandra I. Ramírez-González ${ }^{9}$ and Diana Sanzón-Gómez ${ }^{10}$
}

check for

updates

Citation: Wong-Villarreal, A.;

Méndez-Santiago, E.W.;

Gómez-Rodríguez, O.;

Aguilar-Marcelino, L.; García, D.C.;

García-Maldonado, J.Q.;

Hernández-Velázquez, V.M.;

Yañez-Ocampo, G.;

Espinosa-Zaragoza, S.;

Ramírez-González, S.I.; et al.

Nematicidal Activity of the

Endophyte Serratia ureilytica against

Nacobbus aberrans in Chili Plants

(Capsicum annuum L.) and

Identification of Genes Related to Biological Control. Plants 2021, 10, 2655. https://doi.org/10.3390/ plants10122655

Academic Editors: Konstantin V. Kiselev and Olga A. Aleynova

Received: 12 October 2021

Accepted: 25 November 2021

Published: 3 December 2021

Publisher's Note: MDPI stays neutral with regard to jurisdictional claims in published maps and institutional affiliations.

Copyright: (c) 2021 by the authors. Licensee MDPI, Basel, Switzerland. This article is an open access article distributed under the terms and conditions of the Creative Commons Attribution (CC BY) license (https:/ / creativecommons.org/licenses/by/ $4.0 /)$.
1 División Agroalimentaria, Universidad Tecnológica de la Selva, Ocosingo 29950, Mexico

2 Centro de Investigación en Biotecnología, Universidad Autónoma del Estado de Morelos, Cuernavaca 62209, Mexico; Erick_williams.1994@hotmail.com (E.W.M.-S.); vmanuelh@uaem.mx (V.M.H.-V.)

3 Programa de Fitopatología, Colegio de Postgraduados-Campus Montecillo, Texcoco 56230, Mexico

4 Centro Nacional de Investigación Disciplinaria en Salud Animal e Inocuidad, INIFAP, Jiutepec 62550, Mexico; aguilar.liliana@inifap.gob.mx

5 Red de Manejo Biorracional de Plagas y Vectores, Instituto de Ecología, A.C.—INECOL, Xalapa 91073, Mexico; daniel.cerqueda@inecol.mx

6 Centro de Investigación y de Estudios Avanzados del Instituto Politécnico Nacional, Unidad Mérida, Mérida 97310, Mexico; jose.garcia@cinvestav.mx

7 Laboratorio de Edafología y Ambiente, Universidad Autónoma del Estado de México, Toluca 50000, Mexico; yanezg0206@gmail.com

8 Facultad de Ciencias Agrícolas, Universidad Autónoma de Chiapas, Huehuetán 30660, Mexico; saulez1@gmail.com

9 Laboratorio de Agrotecnologías, Centro Universidad Empresa, Universidad Autónoma de Chiapas, Tuxtla Gutiérrez 29050, Mexico; sanir@yahoo.com

10 División Ciencias de la Vida, Departamento de Agronomía, Campus Irapuato-Salamanca, Universidad de Guanajuato, Irapuato 36500, Mexico; dianas7@yahoo.com.mx

* Correspondence: wova79@hotmail.com (A.W.-V.); olgago@colpos.mx (O.G.-R.)

\begin{abstract}
The genus Serratia is widely distributed in soil, water, plants, animals, invertebrates, and humans. Some species of this genus have antifungal, antibacterial, and nematicidal activity. In this work, the nematicidal activity of the endophytic strain of Serratia sp. in chili, Capsicum annuum L., is reported, where at a bacterial concentration of $4 \times 10^{9} \mathrm{cel} / \mathrm{mL}$, the penetration of nematodes into the roots significantly decreased by 91 and $55 \%$ at 7 and 21 days after inoculation. This bacterial concentration also significantly decreased the number of galls, eggs, egg masses and reproduction factor produced by Nacobbus aberrans in Chili plants, with respect to the control where this bacterial strain was not applied. In the analysis of the genome of the strain, based on average nucleotide identity (ANI), the isolate could be affiliated to the species Serratia ureilytica. The size of the genome is $5.4 \mathrm{Mb}$, with a $59.3 \%$ content of GC. Genes related to the synthesis of chitinases, siderophores, proteases $C$, serralisins, hemolysin, and serrawettin W2 that have been reported for biocontrol of nematodes were identified in the genome. It is the first report of Serratia ureilytica with nematicidal activity. Based on these results of nematicidal activity, this strain can be evaluated in the field as an alternative in the biocontrol of Nacobbus aberrans in chili cultivation.
\end{abstract}

Keywords: biocontrol; rhizobacteria; false root knot; vegetables

\section{Introduction}

The false root-knot nematode Nacobbus aberrans is a species located within the top 10 phytoparasitic nematodes worldwide [1], and within the most economically important groups in Mexico in the crops of chili, tomato, and bean [2]. When feeding, the sedentary 
females of N. aberrans induce the formation of galls in the roots, which block the vascular system resulting in a nutritional imbalance and poor water absorption, impacting on crop production [3,4]. In Mexico, losses caused by $N$. aberrans are reported in tomato yields of $55 \%$, chili $50-70 \%$, and beans $36 \%$ [2-5]. In general, chemical control of phytonematodes has been the main management strategy, but this generates contamination problems due to its persistence in the environment, causing animal and human health problems. Additionally, damage to beneficial organisms has been observed [6,7]. Therefore, in recent decades nematode management has been focused on the use of different control methods, such as biocontrol that uses different enemies or natural antagonists such as fungi and bacteria [6].

Numerous species of soil bacteria located in the rhizosphere stimulate plant growth and reduce the nematode population through antagonistic behavior; collectively they are known as plant growth-promoting rhizobacteria (PGPR), among which are the genera Bacillus, Pseudomonas, and Serratia $[8,9]$. Bacteria-nematode interaction can reduce nematode populations by the effect of enzymes, toxins, and secondary metabolites as products of antagonist rhizobacteria. Hydrolytic enzymes, mainly proteases, collagenases, and chitinases, have been proposed to have nematicidal activity due to the degradation of nematode components in their different stages of development [9]. Among the first works in which the use of the Serratia bacteria is reported is that of Mercer et al. [10], who used chitinases produced by Serratia spp. with a deleterious effect on Meloidogyne hapla eggs. Currently, nematicidal activity of Serratia spp. has been reported both under in vitro and in vivo conditions against some plant-parasitic nematodes. Zhao et al. [11] used bacteria of the Pseudomonas, Bacillus, and Serratia genera against Meloidogyne incognita eggs under in vitro conditions and reported the highest larvicidal and ovicidal effect of Serratia proteamaculans with $99.1 \%$ mortality of second stage juveniles (J2) and $61.11 \%$ egg mortality. Paiva et al. [12] report nematicidal activity of Serratia sp. against Bursaphelenchus spp. by attributing the presence of proteases in the supernatant. On the other hand, Almaghrabi et al. [8] evaluated the effect of six PGPRs: Pseudomonas putida, P. fluorescens, S. marcescens, B. amyloliquefaciens, B. subtilis, and B. cereus on the growth of tomato plants as well as against the root-knot nematode $M$. incognita, where the highest dry weight, height, and number of fruits per plant were obtained in plants treated with the bacterium $S$. marcescens, which corresponded to the lowest number of J2 in the soil, galls, and eggs masses per root. Similarly, the prodigiosin pigment extracted from S. marcescens has been used with a nematicidal effect on the hatching of the J2 from the egg of the PPN Radopholus similis and M. javanica [13]. Abebe-Akele et al. [14] reported the genomes of Serratia marcescens DB11, S. proteamaculans 568, and Serratia sp., isolated from the nematode Caenorhabditis briggsae, where they identified genes that are related to entomopathogenic activity against Galleria mellonella larvae. The genome of Serratia sp. YD25, isolated from the rhizosphere of tobacco cultivation, bore genes related to the production of serrawettin $\mathrm{W} 2$ and prodigiosin metabolites important for biocontrol [15].

According to these antecedents, the nematicidal activity of Serratia sp., isolated from nodules of Mimosa pudica from the state of Chiapas, Mexico, was evaluated in vitro. A mortality of $88.8 \%$ was recorded in juveniles of the second stage of N. aberrans, and $100 \%$ in infective larvae (L3) of the sheep parasitic nematode Haemonchus contortus and free-living Panagrellus redivivus [16]. Therefore, the present research aims to report the genome of a strain of Serratia with nematicidal activity against N. aberrans in chili plants, for the identification of genes associated with this activity and contribute to the sustainable management of this phytoparasitic nematode given its economic impact on protected agriculture.

\section{Results}

\subsection{Nematode Penetration}

The percentage of nematodes that penetrated the root of each plant at 7 and 21 days after inoculation (dai) with N. aberrans is presented in Figure 1A,B. The plants with the highest percentage of nematode penetration on both evaluation dates were recorded in the treatment where only the nematode $(\mathrm{Na})$ was inoculated, and with significant differences 
$(p<0.05)$ with respect to the different treatments evaluated. At 7 days, no significant differences were recorded between the treatments of the plants treated with nematicide, the supernatant, and Serratia sp. with the two lowest concentrations (Se-2 and Se-3 treatments with $2 \times 10^{9}$ and $3 \times 10^{9} \mathrm{cel} / \mathrm{mL}$, respectively). The aforementioned treatments (iu, supernatant, Se-2, and Se-3) presented a significant reduction $(p<0.05)$ with respect to the Na treatment between 53 to $65 \%$, and for the highest concentration $\left(\mathrm{Se}-4,4 \times 10^{9} \mathrm{cel} / \mathrm{mL}\right)$ with a value of $91 \%$. This last treatment (Se-4) had the lowest penetration percentage and significantly different from the evaluated treatments. At 21 dai there was a similar behavior to 7 dai, except for the Se-3 treatment, which presented a significantly $(p<0.05)$ lower percentage of nematodes that penetrated with respect to the plants treated with the supernatant, and without differences with respect to at the highest concentration of the bacterium Se-4 $\left(4 \times 10^{9} \mathrm{cel} / \mathrm{mL}\right)$. The treatments in which the plants were treated with nematicide, supernatant, and the lowest concentration of the bacteria registered a significant reduction $(p<0.05)$ compared to the control $(\mathrm{Na})$ from 28 to $45 \%$, and for the treatments with Serratia (Se-3, and Se-4) with reductions of 45 and 55\%. In general, the lowest percentage of nematodes was registered in the Se- 4 treatment with a reduction of $91 \%$ at 7 dai and $55 \%$ at 21 dai with respect to the Control (Na).
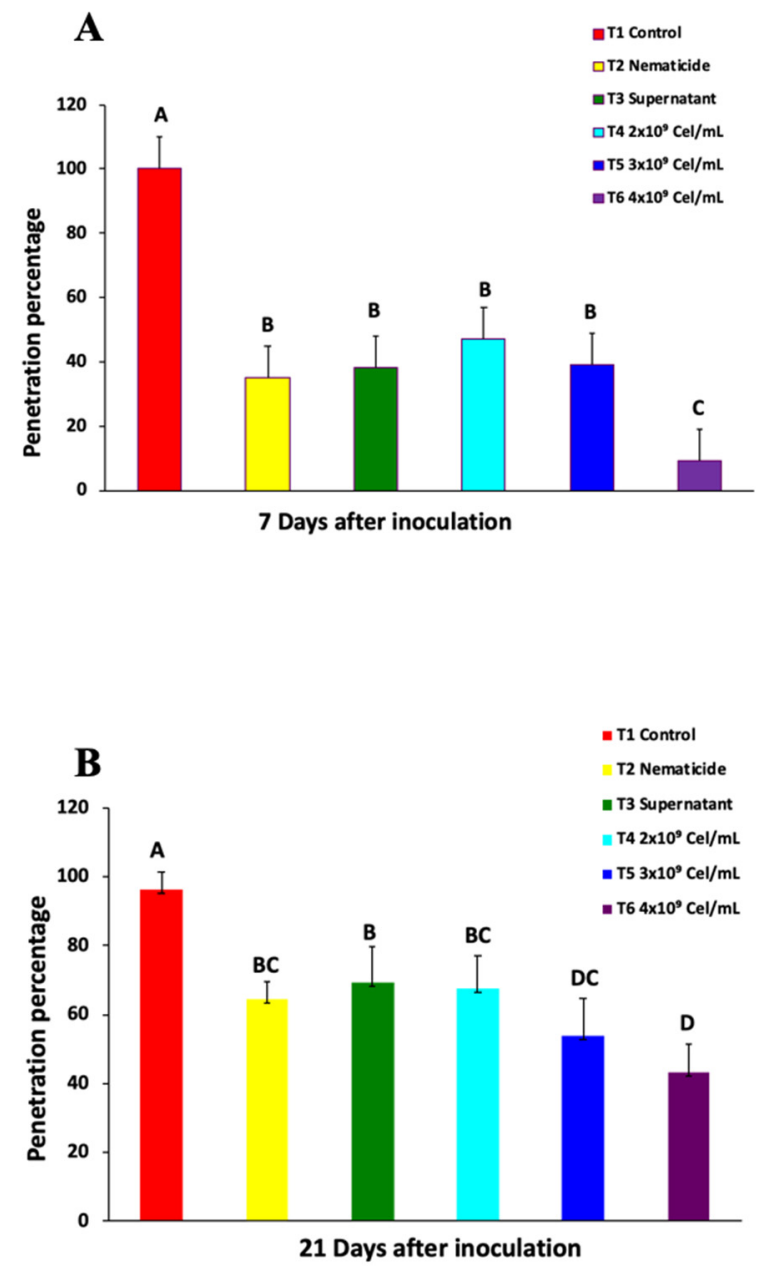

Figure 1. Penetration percentage of nematodes per root in chili plants inoculated with Nacobbus aberrans (A) At 7 days after inoculation. Coefficient Variation $=8.92 \%$. (B) At 21 days after inoculation. Coefficient Variation $=6.96 \%$. Treatments with the same letter do not have significant differences.

\subsection{Galls, Egg Masses, Eggs, and Reproduction Factor}

The number of galls, egg masses, eggs, and reproduction factor are presented in Table 1. The control $(\mathrm{Na})$ was significantly $(p<0.05)$ different, presenting the highest 
number of galls with respect to the plants treated with the nematicide and the concentration of $4 \times 10^{9} \mathrm{cel} / \mathrm{mL}$ of Serratia sp. (Se-4), as is evident in Figure 2, greater number of egg masses and reproduction factor with respect to the lower and higher concentration of the bacteria $2 \times 10^{9} \mathrm{cel} / \mathrm{mL}$, Se-2 and $4 \times 10^{9} \mathrm{cel} / \mathrm{mL} \mathrm{Se}-4$, and the highest number of eggs with respect to all the treatments evaluated (Table 1). Treatment Se-4 registered the lowest number of galls, masses of eggs, and eggs with reductions of $47 \%, 62 \%$, and $91 \%$, respectively, with respect to the control $(\mathrm{Na})$ when considering this treatment $(\mathrm{Na})$ with a value of $100 \%$, and without significant differences with respect to the application of the nematicide. Furthermore, the Se-4 treatment had the lowest reproduction factor, equivalent to 1.26 , while in the control it was 3.3 (Table 1).

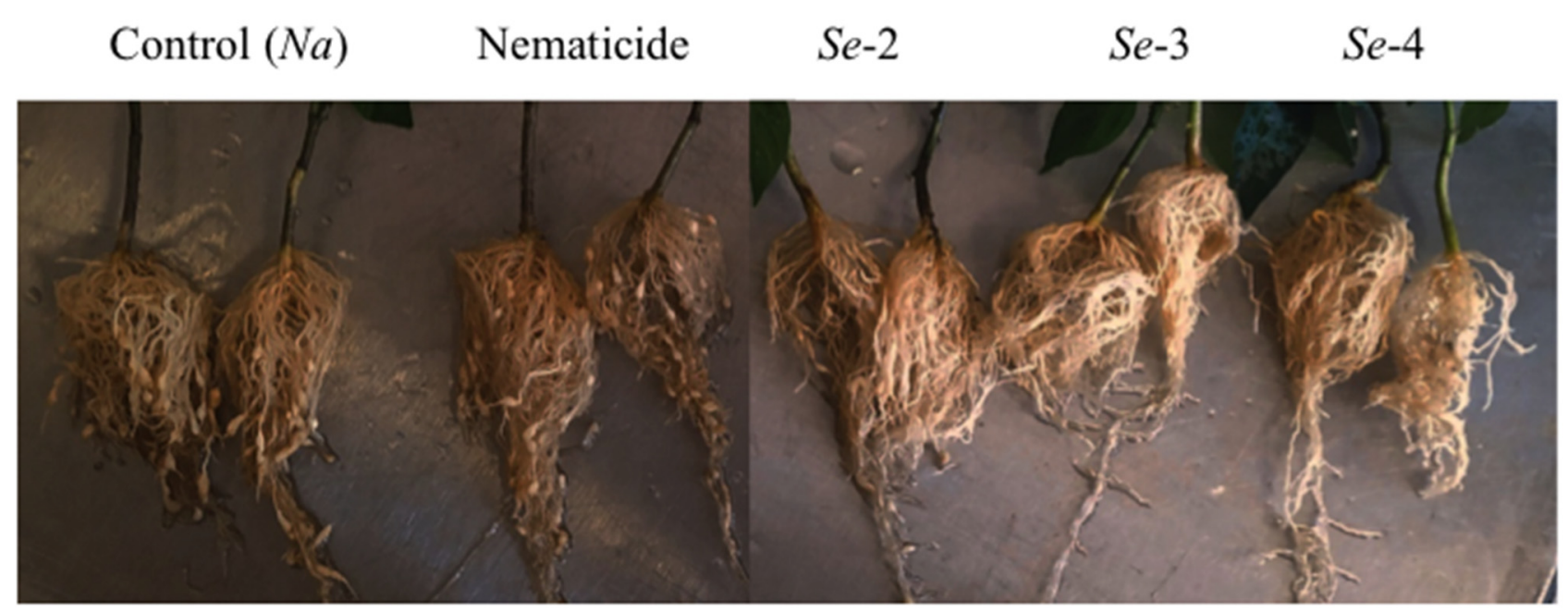

Figure 2. Galling in chili plants cv. Tampiqueño 42 days after inoculation with $1000 \mathrm{~J} 2 \mathrm{of}$ N. aberrans in the growth chamber. $\mathrm{Na}$, plants inoculated only with the nematode; Nematicide, plants with Nemacur ${ }^{\circledR}$; plants with the bacteria at different concentrations: Se-2 $\left(2 \times 10^{9} \mathrm{cel} / \mathrm{mL}\right)$, Se-3 $\left(3 \times 10^{9} \mathrm{cel} / \mathrm{mL}\right)$, and Se-4 $\left(4 \times 10^{9} \mathrm{cel} / \mathrm{mL}\right)$.

Table 1. Number of galls, egg masses, eggs/g of root, and reproduction factor (RF) in chili plants cv. Tampiqueño 42 days after inoculation with $1000 \mathrm{~J} 2$ of N. aberrans in the growth chamber.

\begin{tabular}{ccccc}
\hline Treatments & Galls & Egg Masses & Eggs & RF (\%) \\
\hline T1 & $41.0 \pm 1.4 \mathrm{a}$ & $16 \pm 1.6 \mathrm{a}$ & $584 \pm 60 \mathrm{a}$ & $3.31 \pm 0.45 \mathrm{a}$ \\
T2 & $22.5 \pm 1.1 \mathrm{~b}$ & $9 \pm 1.3 \mathrm{ab}$ & $145 \pm 12.6 \mathrm{~cd}$ & $2.28 \pm 0.64 \mathrm{ab}$ \\
T3 & $29.0 \pm 0.9 \mathrm{ab}$ & $9 \pm 1.1 \mathrm{ab}$ & $168 \pm 18.8 \mathrm{~b}$ & $2.29 \pm 0.56 \mathrm{ab}$ \\
T4 & $25.5 \pm 1.1 \mathrm{ab}$ & $7 \pm 1.2 \mathrm{~b}$ & $151 \pm 16.8 \mathrm{bcd}$ & $1.75 \pm 0.57 \mathrm{~b}$ \\
T5 & $27.1 \pm 0.8 \mathrm{ab}$ & $8 \pm 0.5 \mathrm{ab}$ & $161 \pm 19.6 \mathrm{bc}$ & $1.87 \pm 0.38 \mathrm{ab}$ \\
T6 & $21.6 \pm 1.0 \mathrm{~b}$ & $6 \pm 0.8 \mathrm{~b}$ & $51 \pm 7.9 \mathrm{~d}$ & $1.26 \pm 0.41 \mathrm{~b}$ \\
CV $(\%)$ & 16.7 & 25.1 & 26.8 & 25.1 \\
\hline
\end{tabular}

$\overline{\mathrm{RF}}=$ (number of eggs per root/1000 J2) (100). $n=10$. Values with the same letter are not significantly different, Tukey, $(p<0.05) . \mathrm{CV}=$ Coefficient Variation.

\subsection{Genome Assembly and Annotation}

According to the average nucleotide identity (ANI) analysis of the genome of strain UTS and closely related genomes from GenBank, the highest ANI value was $98.96 \%$ with Serratia ureilytica Niva51(GCA_013375155.1). This value is higher than the widely accepted threshold range (95-96\%) for species demarcation [17,18]. The Serratia ureilytica UTS strain contains 134 contigs with a size of $5.40 \mathrm{Mbp}$ (Table 2); the genome includes 5303 coding sequences (CDS) and 93 RNA genes. The mean GC content is $59.3 \%$, similar to $S$. marcescens strains; 954 hypothetical proteins and 4394 proteins with assigned functions were also reported (Table 2). 
Table 2. Genome features of Serratia ureilytica UTS.

\begin{tabular}{cc}
\hline Features & Chromosome \\
\hline Contigs & 134 \\
Genome size & $5,401,403 \mathrm{bp}$ \\
GC content $(\%)$ & 59.32561 \\
Coding gene & 5303 \\
tRNA & 87 \\
rRNA & 6 \\
Hypothetical proteins & 954 \\
Proteins with functional assignments & 4349 \\
\hline
\end{tabular}

Genome sequence analysis revealed the presence of homologous proteins involved in the biosynthesis of bacteriocin pyoverdin, colicin $\mathrm{V}$, hydrogen cyanide, hemolysin, serrawettin W2, proteases, chitinases A, chitinases B and chitinases C (Figure 3, Table 3). The results of the enzyme activity assay also indicated that $S$. ureilytica UTS possesses the ability to secrete extracellular protease and chitinase (Figure 4).

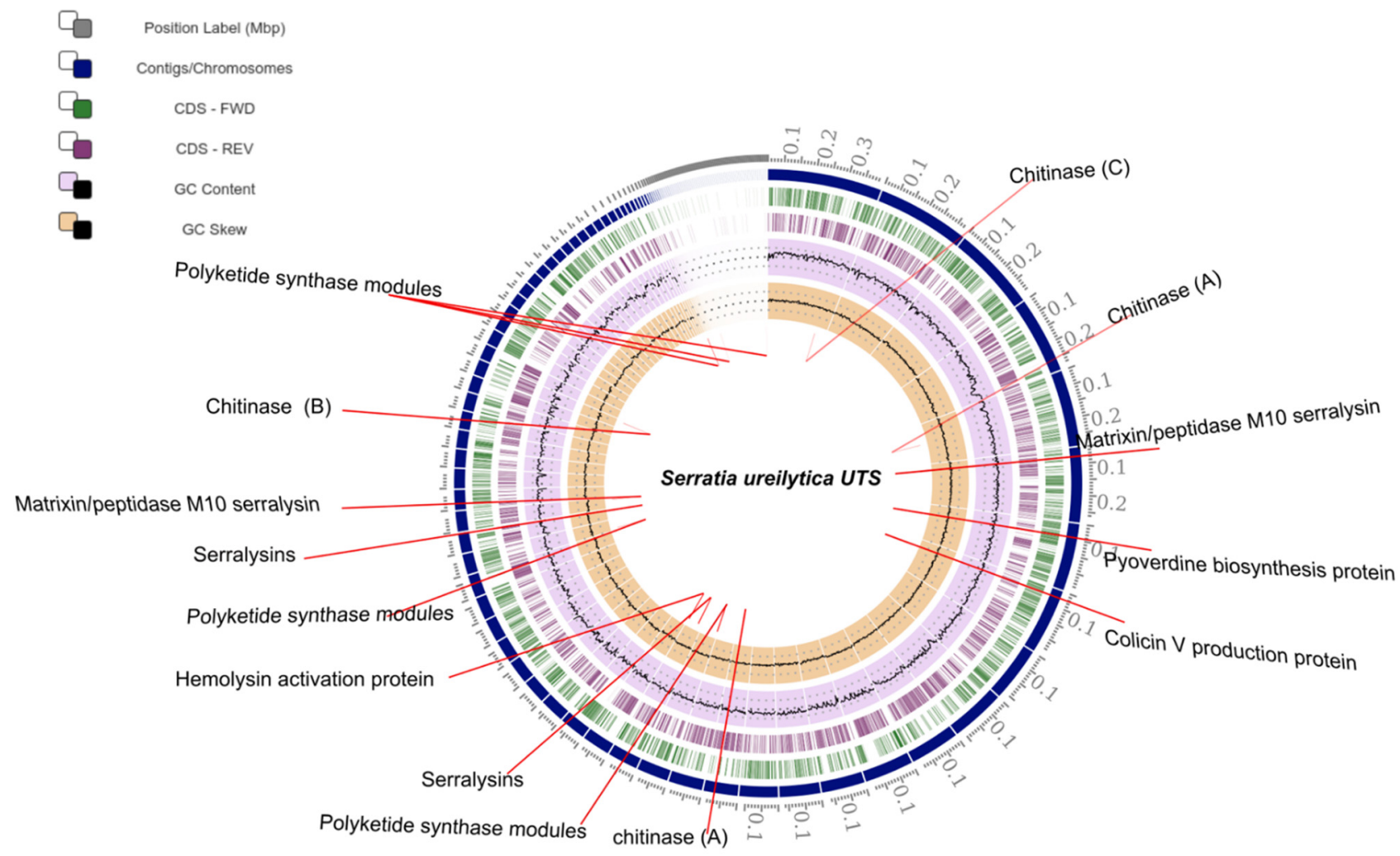

Figure 3. Circular representation of the genome of Serratia ureilytica UTS. From outside to inside: the first circle shows the contigs of the genome; the second and third circles show genes in the forward and reverse strands, respectively; the fourth and fifth circles, GC content and GC skew. 
Table 3. Location of genes and gene products involved in nematicidal activity present in the genome of Serratia ureilytica UTS.

\begin{tabular}{cl}
\hline Genomic Location & \multicolumn{1}{c}{ Serratia ureilytica UTS Protein } \\
\hline $1-6213$ & \\
$87709-93531$ & \\
$3-2393$ & Putative peptide synthetase, containing \\
$1-408$ & non-ribosomal peptide synthetase \\
$3-206$ & \\
$117538-118815$ & Chitinase (A) \\
$76512-78203$ & Chitinase (A) \\
$7930-9444$ & Serine 3-dehydrogenase \\
$56070-57587$ & Colicin V production protein \\
$64025-64888$ & Pyoverdine biosynthesis protein \\
$38269-39687$ & Serine 3-dehydrogenase \\
$36474-38090$ & Protease C \\
$50375-51874$ & Chitinase (B) \\
$49385-50767$ & Matrixin/peptidase M10 serralysin \\
$58625-59740$ & Chitinase (C) \\
$23187-28013$ & Hemolysin \\
$28059-29738$ & Hemolysin activation protein \\
$7930-9444$ & Serralysin \\
\hline
\end{tabular}
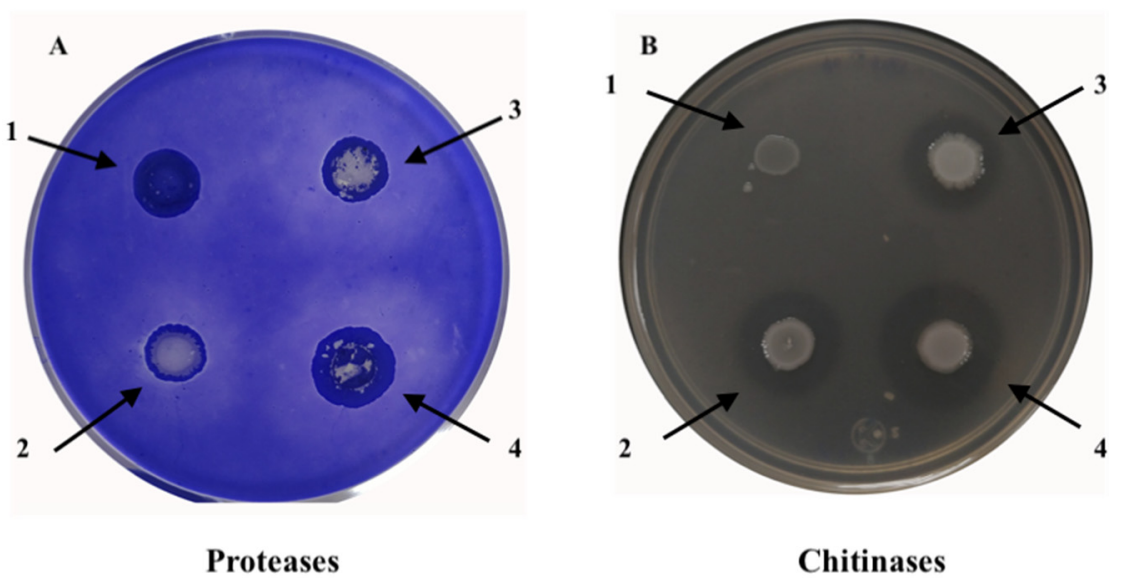

Proteases

\section{Chitinases}

Figure 4. (A) Secretion of extracellular protease and (B) chitinase activity from Serratia ureilytica UTS. The culture medium used for the detection of extracellular protease contains skim milk and for chitinase it contains $1 \%$ colloidal chitin, respectively. The protease or chitinase activity is observed by the formation of halo around the bacterial growth after incubation for $16-24 \mathrm{~h}$ at $30{ }^{\circ} \mathrm{C}$. The inoculated spots in the culture medium correspond to different optical densities quantified at $600 \mathrm{~nm}$ of the culture of Serratia ureilytica UTS 2: $(0.4), 3(0.6)$ and $4(0.8)$, the inoculated point that does not present halo, corresponds to the control negative, (1) Escherichia coli.

\subsection{Protease and Chitinase Degradation Assays}

Chitinase activity was detected by observing the formation of halos around S. ureilytica UTS in the medium with colloidal chitin; Protease activity was observed by the formation of halos in a medium containing skim milk. In Figure 4 different sizes of halos can be observed both in chitinase and protease activity, this is due to the fact that different optical densities of the culture were inoculated: $1(0.4), 2$ (0.6) and $3(0.8)$; in the inoculum where no halo is observed is the negative control: 4 (Escherichia coli)

\subsection{Figures and Tables}

(T1) Plants only with the nematode (Na), (T2) Plants treated with commercial nematicide (Nematicide), (T3) Plants treated with the bacterial growth supernatant (Supernatant) (T4), Plants treated with Serratia sp. (Se-2, $\left.2 \times 10^{9} \mathrm{cel} / \mathrm{mL}\right),(\mathrm{T} 5)$ Plants treated with Serratia 
sp. $\left(\right.$ Se-3, $\left.3 \times 10^{9} \mathrm{cel} / \mathrm{mL}\right)$, and (T6) Plants treated with Serratia sp. (Se-4, $\left.4 \times 10^{9} \mathrm{cel} / \mathrm{mL}\right)$. The commercial nematicide used was Nemacur ${ }^{\circledR} 38.80 \%$ CE (Fenamiphos) applied at a dose of $2 \%$. The values (mean \pm SD) in each column followed by the same letter are not significantly different.

\section{Discussion}

The application of the bacteria Serratia sp. to the inoculation with the juveniles of the second stage (J2) of $N$. aberrans in chili seedlings showed a nematicidal effect at the highest concentration evaluated (Se-4, $4 \times 10^{9} \mathrm{cel} / \mathrm{mL}$ ) (Figure 1). It presented significant differences $(p<0.05)$ both in the percentage of nematodes that penetrated the root and in the number of galls, egg masses, eggs, and reproduction factor with respect to the plants that were inoculated only with the nematode ( $\mathrm{Na}$, Control) (Table 1, Figure 1). In the assessment of the nematicidal capacity of this strain of bacteria under in vitro conditions, an $88.8 \%$ mortality of J2 of $N$. aberrans was recorded [16], an effect confirmed in vivo under the conditions evaluated in the present investigation. The species Serratia is reported as an efficient biological chitin degrading bacterium [19]. Within the characterization of the strain used, chitinase production was recorded (specific activity of 13,999 U/mg) [16]. The main component of the nematode cuticle is collagen, without the presence of chitin [20-22]; however, there are reports that indicate a nematicidal effect of chitinases both in solution and those produced by different species of bacteria, among which is Serratia sp. when using a chitinase solution. Millew and Sands [23] observed structural changes in the cuticle of juveniles (J2) of Tylenchorhynchus dubius incubated after $2.5 \mathrm{~h}$, and a reduction in the movement of individuals above 75\%. Likewise, Sánchez [24] used a chitosan solution at concentrations of 2000 and $1500 \mathrm{ppm}$ and recorded a mean immobilization of 145.9 and 140.5 in juveniles of the second stage (J2) of N. aberrans at $72 \mathrm{~h}$, respectively, compared to a mean of 51.9 when using water. The use of a chitinase solution can be comparable to when the supernatant in which the bacteria grew was used, a treatment in which a significantly $(p<0.05)$ lower percentage of nematodes that penetrated the chili root was recorded at 7 and 21 dai (9.9 and 6.9\%, respectively), and in the number of eggs per gram of root (168) at the end of the evaluation, compared to the control. In relation to the penetration of nematodes at 21 dai, Sánchez [24] also reports a lower percentage of individuals in CM-334 chili roots treated with a chitosan solution at $2000 \mathrm{ppm}$ and inoculated at the same time with $N$. aberrans J2.

The greatest nematicidal effect was evidenced when the highest concentration $\left(4 \times 10^{9} \mathrm{cel} / \mathrm{mL}\right)$ of Serratia was used; This effect is perhaps not only related to the chitinolytic activity of the strain [16], but also to the activity of other enzymes such as proteases.In general, rhizobacteria are attributed a nematicidal effect due to the activity of enzymes such as proteases, collagenases, and chitinases, which can act on different chemical components of nematodes in different stages [9]. Hegazy et al. [25] report a high percentage of juvenile mortality (J2) of M. incognita (96\%) after $24 \mathrm{~h}$ with $S$. marcescens subsp. marcescens due to a high activity of chitinases and proteases, and they inferred that the activity of chitinases was more relevant than proteases in the mortality of nematodes. However, Paiva et al. [12] attributed the death of the nematode B. xylophilus to the production of proteases of Serratia sp., a similar effect was observed in the treatments where the bacteria were used at different concentrations (treatments Se-2, Se-3, and Se-4). The lowest number of nematodes that penetrated the root of the chili plants was recorded, with the treatment with the highest concentration of cel $/ \mathrm{mL}$ of Serratia sp., (Se-4), significant differences were observed $(p<0.05)$ with respect to the treatment where the chemical nematicide is applied (Table 1$)$.

Meanwhile, the use of the nematicide registered a significantly $(p<0.05)$ lower number of galls and eggs with respect to the control (Table 1). In contrast, the use of Serratia sp., $\left(\mathrm{Se}-4,4 \times 10^{9} \mathrm{cel} / \mathrm{mL}\right)$ registered the lowest values of number of galls, egg masses, eggs, and reproduction factor, this last variable with a value of 1.26 and with significant differences $(p<0.05)$ with respect to the control with 3.31 (Table 1). This represents that there will 
be a higher level of inoculum in the rhizosphere of this last treatment. Our results are added to those reported by other studies in which an enzymatic activity of the species Serratia sp. with nematicidal effect against some species of plant-parasitic nematodes are reported. Almaghrabi et al. [8] treated tomato plants with S. marcescens and inoculated with $M$. incognita and recorded a lower number of galls and egg masses. In addition, in tomato, Zhao et al. [11] treated the seeds with $S$. marcescens and observed a lower number of galls and juveniles of M. incognita in the soil. For their part, Zeinat et al. [26] recorded a lower number of galls in Vicia fava plants inoculated with Meloidogyne sp. and treated with S. marcescens. Furthermore, the use of Serratia sp. against $N$. aberrans can be favored by the eating habits of this species of nematode, since it behaves as a migratory endoparasite in the stages of J2, J3, J4 and vermiform immature female when re-entering the root of its hosts, and only establishes itself as a sedentary endoparasite in the female stage, being exposed to the microorganisms of the rhizosphere at various stages within its life cycle $[27,28]$. Meanwhile Meloidogyne sp., which is also a root-knot nematode, penetrates the J2 to the root and establishes itself in it [28]. With the same population of N. aberrans that was used in the present study, Cristóbal et al. [29] point out that juveniles J3 and J4 survive in the soil under field conditions and without hosts for 12 months, conserving their infectivity when inoculated in tomato plant roots. This condition makes them vulnerable to the enzymatic activity of different species of bacteria and fungi. The nematicidal activity of this strain in chili plants generated interest in identifying genes that may be involved in the biosynthesis of metabolites involved in biocontrol.

Genome analysis allowed the strain to be identified as S. ureilytica with $98.93 \%$ identity, based on average nucleotide identity (ANI), this strain does not have a plasmid; However, strains such as S. ureilytica CC119 (PRJNA487218) and S. ureilytica JBIWA004 (PRJNA725976) have been reported that possess plasmid. Some strains of Serratia produce metabolite prodigiosin related to nematicidal activity against juvenile states of Radopholus similis and M. javanica, as well as egg hatching [15-28]. However, in this strain this pigment is not present, so the nematicidal activity must be related to the production of chitininase enzymes as reported by Hegazy et al. [25], where the activity of this enzyme produced by S. marcescens subsp. marcescens has $96 \%$ mortality in juveniles (J2) of the nematode M. incognita.

Another molecule that is associated with nematicidal activity is Serrawettin W2, a biosurfactant of lipopetidic nature [30]. The genes that encode for this molecule have been identified in S. ureilytica UTS (Table 3), this agrees with that reported by MarquesPereira et al. [31], where they identified these genes in different Serratia species. Genes associated with the production of protease enzymes in the genome were also identified. These enzymes have been detected in Serratia sp. A88copa13, where they were related to nematicidal activity against Bursaphelenchus tusciae, B. mucronatus, and B. conicaudatus nematodes (Paiva et al. [12]. Although the purification of proteases and chitinases in the strain of S. ureilytica TKU013 have been reported for the degradation of squid pen; as well as the production of Serratiw w2 in other strains of S. ureilytica [32]. No reports of $S$. ureilytica with nematicidal activity were found, so it is considered that the S. ureilytica UTS strain is the first report of this species with nematicidal activity.

\section{Materials and Methods}

\subsection{Plant Material}

Chili type serrano cv. Tampiqueño, susceptible to $N$. aberrans, was used. The chili seeds were disinfected with $0.5 \%$ sodium hypochlorite for $1 \mathrm{~min}$ and rinsed with sterile distilled water. For their germination, they were placed on sterile paper towels moistened with sterile distilled water in germinating boxes at $28^{\circ} \mathrm{C}$. The seedlings were transplanted into pots with $25 \mathrm{cc}$ of sterile fine sand (one seedling per pot), and kept in a growth chamber at a temperature of $27 \pm 1{ }^{\circ} \mathrm{C}$, with a photoperiod of $14 \mathrm{~h}$ with a light intensity of 6768 lux (Fluorescent light). It was fertilized weekly with a nutrient solution (3.15 g of Nitrofoska ${ }^{\circledR}$ 12-12-12-2 per liter of sterile water). 


\subsection{Obtaining and Inoculation of Serratia sp. (Se)}

The preparation of the inoculum of the Serratia strain was carried out in PY liquid culture medium containing the following ingredients $(\mathrm{g} / \mathrm{L})$ : tryptone casein (5), yeast extract (3), calcium chloride (0.1) and with the following growth conditions: $30^{\circ} \mathrm{C}, 200 \times \mathrm{rpm}$ for $72 \mathrm{~h}$. The concentrations of each inoculum were adjusted to an optical density (OD) of $0.5\left(2 \times 10^{9} \mathrm{cel} / \mathrm{mL}\right), 0.75\left(3 \times 10^{9} \mathrm{cel} / \mathrm{mL}\right)$, and $1\left(4 \times 10^{9} \mathrm{cel} / \mathrm{mL}\right)$ at $600 \mathrm{~nm}$. The chili plants were inoculated with each concentration of the bacteria 21 days after transplantation and a second inoculation four days after the first.

\subsection{Obtaining Inoculum and Inoculation with N. aberrans ( $\mathrm{Na}$ )}

The inoculum of $N$. aberrans was obtained from galled roots of tomato grown in naturally infested soil at Colegio de Postgraduados, Montecillo campus, State of Mexico, Mexico, and kept in greenhouse tomato plants (cv. Rio Grande). Nematode eggs extraction was carried out following the methodology described by Vrain [33], and they were incubated at $28 \pm 1{ }^{\circ} \mathrm{C}$ in Petri dishes with sterile distilled water, until hatching of the second stage juveniles (J2). Each chili plant with two to three pairs of true leaves was inoculated with $1000 \mathrm{~J} 2$ of N. aberrans five days after inoculation with Serratia sp.

\subsection{Experiment Establishment and Evaluation}

The experiment was established during 2018 and 2019. The experimental design was completely randomized and consisted of six treatments with 22 pots/treatment. Treatments: (1) Na (Control, plants only with the nematode), (2) Nematicide (plants treated with nematicide), (3) Supernatant [plants treated with the supernatant of the liquid culture medium PY (g/L: casein tryptone, 5; yeast extract, 3; and calcium chloride, 0.1), where the bacteria grew], (4) Se-2 (plants treated with Se at an optical density (OD) of 0.5 with a total of $2 \times 10^{9} \mathrm{cel} / \mathrm{mL}$ ), (5) Se-3 (plants treated with Se at an OD of 0.75 with $3 \times 10^{9} \mathrm{cel} / \mathrm{mL}$ ), and 6) Se-4 (plants treated with Se at an OD of 1.0 with $4 \times 10^{9} \mathrm{cel} / \mathrm{mL}$ ). The commercial nematicide used was Nemacur ${ }^{\circledR} 38.80 \%$ CE (Fenamiphos) applied at a dose of $2 \%$.

The nematicidal activity of Serratia sp. On N. aberrans was evaluated 7, 21, and 42 days after inoculation (dai) with the nematode. At 7 and 21 dai, the number of individuals per root of each plant was counted under light microscopy in six plants per treatment, and the results were expressed as a percentage. Plants roots were stained using the sodium hypochlorite-acid fuchsin method [34]. The number of individuals per root was counted using a stereoscopic microscope $(4 \times, 10 \times)$.

At 42 dai of the nematode, the number of galls, egg masses, and eggs/g of root in 10 plants per treatment were counted. In addition, with the number of eggs of each root, the reproduction factor $(\mathrm{RF})$ was calculated where $\mathrm{RF}=$ (Final population/initial population) (100). Egg extraction was carried out according to Vrain [33]. To quantify the egg masses, the roots were immersed in a solution of Phloxin B $(0.15 \mathrm{~g} / \mathrm{L}$ of water) for $20 \mathrm{~min}$ so that the egg masses were stained red and counting was facilitated.

\subsection{Statistical Analysis}

The data on the percentage of penetration of nematodes, number of galls, egg masses, eggs/g of root, and reproduction factor were subjected to an analysis of variance (ANOVA) using the general linear model, and a comparison of means with the Tukey's test and the hovtest to see the homogeneity in the treatments with a significance level of $p \leq 0.05$, with the use of the "Statistical Analysis System" program (SAS version 9.0).

\subsection{DNA Extraction, Library Preparation, and Sequencing}

Genomic DNA was extracted from freshly cultivated cells of the strain using the ZR Fungal/Bacterial DNA Kit kitTM, according to the manufacturer's instructions. DNA was quantified with a Qubit" 3.0 Fluorometer using the Qubit dsDNA HS Assay Kit (Life Technologies, Carlsbad, CA, USA). The library was created from 1 ng of DNA using the Nextera XT DNA Library Prep Kit (Illumina, San Diego, CA, USA), and purified 
using AMPure XP magnetic beads, according to the supplied protocol (Beckman Coulter Genomics, Brea, CA, USA). The correct size of the library was verified on an Advanced QIAxcel (QIAGEN, Germantown, TN, USA). Paired-end sequencing $(2 \times 150 \mathrm{bp})$ was performed at CINVESTAV Mérida with the MiSeq platform (Illumina, San Diego, CA, USA) using a 300-cycle MiSeq Reagent Micro Kit v2. Raw sequence data produced in this study was deposited in NCBI under the Bioproject accession number PRJNA759051.

\subsection{Genome Assembly and Annotation and Taxonomic Classification}

The paired-end $(2 \times 150)$ raw Illumina reads were trimmed for adapters and filtered at a Q score $>30$. De novo assembly was performed with SPAdes (v. 3.12) [35]. Assembly was exported to the PATRIC platform [36], and annotation was performed with RAST (Rapid Annotations using Subsystems Technology) [37]. Taxonomic identification was performed with the GTDB-Tk software, which uses a set of 120 marker genes to place the draft genome within the curated Genome Taxonomy Database references tree and FastANI (Average Nucleotide Identity) [38].

\subsection{Homologous Identification}

A reciprocal blast between amino acid sequences of the Serratia ureilytica UTS draft genome and the amino acid sequences of Serratia marcescens complete genome (MJVB00000000, MJVC00000000) was performed using blastp with a threshold of $90 \%$ of similarity and e-value $<0.00001$.

\subsection{Accession of the Genome Sequences}

The data from this Whole Genome Shotgun project was deposited in GenBank with Accession JAIQCT000000000, Bioproject PRJNA759051 and Biosample access number SAMN21162916

\subsection{Protease and Chitinase Degradation Assays}

The bacteria Serratia strain was seeded in triplicate in solid NBRIP (glucose, 1\%; $\mathrm{Ca}_{3}\left(\mathrm{PO}_{4}\right)_{2}, 0.5 \% ;\left(\mathrm{NH}_{4}\right)_{2} \mathrm{SO}_{4}, 0.01 \% ; \mathrm{MgSO}_{4} \cdot 7 \mathrm{H}_{2} \mathrm{O}, 0.025 \% ; \mathrm{KCl}, 0.02 \% ; \mathrm{MgCl}_{2} \cdot 6 \mathrm{H}_{2} \mathrm{O}$, $0.5 \%$; Congo red, $2.5 \mathrm{mg} / \mathrm{mL}$; agar, $1.8 \%$ ) culture medium supplemented with $1 \%(w / v)$ of colloidal chitin (Sigma-Aldrich Corp, Darmstadt, Alemania.) as sole carbon sources [16]. The bacteria isolates were checked for their production of chitinase by observation of clear zone around the colonies after four days incubation at $30{ }^{\circ} \mathrm{C}$. To determine the protease activity, the Serratia strain was seeded in triplicate in the nutritive culture medium supplemented with $1 \%(w / v)$ skimmed milk; the protease activity was observed by the appearance of a clear halo around the bacterial growth [39].

\section{Conclusions}

The S. ureilytica UTS strain used in the present research showed a nematicidal effect against $N$. aberrans in chili plants, type serrano cv. Tampiqueño that were inoculated. The inoculated plants significantly decreased the number of nematodes (J2) in the root, number of galls, egg masses, eggs, and reproduction factor. This nematicidal activity may be related to the genes identified in the genome of the strain that have been reported with biocontrol activity. The use of this bacterial strain has potential in the sustainable management of the false root knot N. aberrans.

Author Contributions: Conceptualization, E.W.M.-S., O.G.-R., L.A.-M. and A.W.-V.; methodology, E.W.M.-S., O.G.-R., L.A.-M. and A.W.-V.; software, D.C.G., J.Q.G.-M. and V.M.H.-V.; formal analysis, V.M.H.-V., G.Y.-O., S.I.R.-G., S.E.-Z., O.G.-R., D.S.-G., L.A.-M. and A.W.-V.; investigation, E.W.M.-S., O.G.-R., L.A.-M. and A.W.-V.; data curation D.C.G. and J.Q.G.-M.; writing-original draft preparation, G.Y.-O., O.G.-R., L.A.-M. and A.W.-V.; writing-review and editing, O.G.-R., L.A.-M., S.I.R.-G., S.E.-Z., D.S.-G. and A.W.-V.; supervision, O.G.-R., L.A.-M. and A.W.-V. All authors have read and agreed to the published version of the manuscript. 
Funding: The present research was partial financed by the National Problems project, Consejo Nacional de Ciencia y Tecnología, Mexico (CONACYT), project number 9342634372.

Institutional Review Board Statement: Not applicable.

Informed Consent Statement: Not applicable.

Data Availability Statement: The data from this Whole Genome Shotgun project was deposited in GenBank under the accession number Bioproject PRJNA759051.

Conflicts of Interest: The authors declare no conflict of interest.

\section{References}

1. Jones, J.T.; Haegeman, A.; Danchin, E.G.J.; Gaur, H.S.; Jones, M.G.K.; Kikuchi, T.; Manzanilla-López, R.; Palomares-Rius, J.E.; Wesemael, W.M.L.; Perry, R.N. Top 10 plant-parasitic nematodes in molecular plant pathology. Mol. Plant Pathol. 2013, 14, 946-961. [CrossRef]

2. Cid del Prado-Vera, I.; Franco-Navarro, F.; Godinez-Vidal, D. Plant parasitic nematodes and management strategies of major crops in Mexico. In Plant Parasitic Nematodes in Sustainable Agriculture of North America, 1st ed.; Subbotin, S.A., Chitambar, J.J., Eds.; Springer: Sacramento, CA, USA, 2018; pp. 31-68. [CrossRef]

3. Nicol, J.M.; Turner, S.J.; Coyne, D.L.; den Nijs, L.; Hockland, S.; Maafi, Z.T. Key nematodes threatening major agricultural crops of importance worldwide. In Genomics and Molecular Genetics of Plant-Nematode Interactions; Jones, J.T., Gheysen, G., Fenoll, C., Eds.; Springer: Dordrecht, Germany, 2011; pp. 21-44. [CrossRef]

4. Castillo, P.; Stanley, J.; Inserra, R.N.; Manzanilla, L.R.H. Pratylenchidae-the lesión nematodes. In Practical Plant Nematology; Manzanilla-López, R.H., Marban-Mendoza, N., Eds.; Biblioteca Básica de Agricultura: Ciudad de Mexico, Mexico, 2012; pp. 411-478.

5. Manzanilla, L.R.H.; Costilla, M.A.; Doucet, M.; Franco, J.; Inserra, R.N.; Lehman, P.S.; Cid del Prado-Vera, I.; Souza, R.M.; Evans, K. The genus Nacobbus Thorne \& Allen, 1944 (Nematoda: Pratylenchidae): Systematics, distribution, biology and management. Nematropica 2002, 32, 149-227.

6. Chen, S.; Dickson, D.W. Biological control of plant-parasitic nematodes. In Practical Plant Nematology; Manzanilla-López, R.H., Marbán-Mendoza, N., Eds.; Biblioteca Básica de Agricultura: Ciudad de Mexico, México, 2012; pp. 761-768.

7. Stirling, G.R. Ecosystem services and the concept of 'Integrated soil biology management'. In Biological Control of Plant-Parasitic Nematodes: Soil Ecosystem Management in Sustainable Agriculture; Stirling, G.R., Ed.; CABI: Wallingford, UK, 2014 ; pp. 3-11.

8. Almaghrabi, O.A.; Massoud, S.I.; Abdelmoneim, T.S. Influence of inoculation with plant growth promoting rhizobacteria (PGPR) on tomato plant growth and nematode reproduction under greenhouse conditions. Saudi J. Biol. Sci. 2013, 20, 57-61. [CrossRef] [PubMed]

9. Castaneda, A.C.; Aballay, E. Rhizobacteria with nematicide aptitude: Enzymes and compounds associated. World J. Microbiol. Biotechnol. 2016, 32, 2-7. [CrossRef]

10. Mercer, C.F.; Greenwood, D.R.; Grant, J.L. Effect of Plant and Microbial Chitinases On the Eggs and Juveniles of Meloidogyne hapla Chitwood (Nematoda: Tylenchida). Nematologica 1992, 38, 227-236. [CrossRef]

11. Zhao, D.; Zhao, H.; Zhao, D.; Zhu, X.; Wang, Y.; Duan, Y.; Xuan, Y.; Chen, L. Isolation and identification of bacteria from rhizosphere soil and their effect on plant growth promotion and root-knot nematode disease. Biol. Control 2018, 119, 12-19. [CrossRef]

12. Paiva, G.; Proença, D.N.; Francisco, R.; Verissimo, P.; Santos, S.S.; Fonseca, L.; Abrantes, I.M.; Morais, P.V. Nematicidal bacteria associated to pinewood nematode produce extracellular proteases. PLoS ONE 2013, 7, e79705. [CrossRef]

13. Price, M.N.; Dehal, P.S.; Arkin, A.P. FastTree 2-approximately maximum-likelihood trees for large alignments. PLoS ONE 2010, 5, e9490. [CrossRef]

14. Abebe-Akele, F.; Tisa, L.S.; Cooper, V.S.; Hatcher, P.J.; Abebe, E.; Thomas, W.K. Genome sequence and comparative analysis of a putative entomopathogenic Serratia isolated from Caenorhabditis briggsae. BMC Genom. 2015, 8, 531. [CrossRef]

15. Su, C.; Liu, Y.; Sun, Y.; Li, Z. Complete genome sequence of Serratia sp. YD25 (KCTC 42987) presenting strong antagonistic activities to various pathogenic fungi and bacteria. J. Biotechnol. 2017, 45, 9-13. [CrossRef]

16. Méndez-Santiago, E.W.; Sánchez-Cruz, R.; Folch Mallol, J.L.; Aguilar-Marcelino, L.; Hernández-Velázquez, V.M.; Gómez-Rodríguez, O.; Villar-Luna, E.; Wong-Villarreal, A. Serratia sp., an endophyte of Mimosa pudica nodules with nematicidal, antifungal activity and growth promoting characteristics. Arch. Microbiol. 2020, 203, 549-559. [CrossRef]

17. Kim, M.; Oh, H.S.; Park, S.C.; Chun, J. Towards a taxonomic coherence between average nucleotide identity and 16S rRNA gene sequence similarity for species demarcation of prokaryotes. Int. J. Syst. Evol. Microbiol. 2014, 64, 346-351. [CrossRef]

18. Richter, M.; Rosselló-Móra, R. Shifting the genomic gold standard for the prokaryotic species definition. Proc. Natl. Acad. Sci. USA 2009, 106, 19126-19131. [CrossRef] [PubMed]

19. Suzuki, K.; Sugawara, N.; Suzuki, M.; Uchiyama, T.; Katouno, F.; Nikaidou, N.; Watanabe, T. Chitinases A, B, and C1 of Serratia marcescens 2170 produced by recombinant Escherichia coli: Enzymatic properties and synergism on chitin degradation. Biosci. Biotechnol. Biochem. 2002, 66, 1075-1083. [CrossRef] 
20. Page, A.P.; Stepek, G.; Winter, A.D.; Pertab, D. Enzymology of the nematode cuticle: A potential drug target? Int. J. Parasitol. Drugs Drug Resist. 2014, 4, 133-141. [CrossRef] [PubMed]

21. Page, A.P.; Johnstone, I.L. The cuticle. In The C. Elegans Research Community; WormBook: Pasadena, CA, USA, 2007. [CrossRef]

22. Veronico, P.; Gray, L.J.; Jones, J.T.; Bazzicalupo, P.; Arbucci, S.; Cortese, M.R.; Di Vito, M.; De Giorgi, C. Nematode chitin synthases: Gene structure, expression and function in Caenorhabditis elegans and the plant parasitic nematode Meloidogyne artiellia. Mol. Genet. Genom. 2001, 266, 28-34. [CrossRef]

23. Millew, P.M.; Sands, D.C. Effects of hydrolytic enzymes on plant parasitic nematodes. J. Nematol. 1977, 9, $192-197$.

24. Sánchez, P.J.F. Efecto de quitina y quitosano sobre huevos y juveniles de nematodos formadores de nodulos radicales, Nacobbus aberrans y Meloidogyne incognita, bajo condiciones in vitro e in vivo. Master's Thesis, Colegio de Postgraduados, Texcoco, México, 2010.

25. Hegazy, M.I.; Salama, A.S.A.; El-Ashry, R.M.; Othman, A.E.I.O. Serratia marcescens and Pseudomonas aeruginosa are promising candidates as biocontrol agents against root-knot nematodes (Meloidogyne spp.). Middle East J. Agricul. Res. 2019, 8, 828-838.

26. Zeinat, K.M.; El-Sayed, S.A.; Radwan, T.E.E.; El-Wahab, G.S.A. Potency evaluation of Serratia marcescens and Pseudomonas fluorescens as biocontrol agents for root-knot nematodes in Egypt. J. Appl. Sci. Res. 2009, 4, 93-102.

27. Eves-van den Akker, S.; Lilley, C.J.; Danchin, E.G.J.; Rancurel, C.; Cock, P.J.A.; Urwin, P.E.; Jones, J.T. The transcriptome of Nacobbus aberrans reveals insights into the evolution of sedentary endoparasitism in plan-parasitic nematodes. Genome Biol. Evol. 2014, 6, 2181-2194. [CrossRef]

28. Perry, R.N.; Moens, M. Introduction to plant-parasitic nematodes; modes of parasitism. In Genomics and Molecular Genetics of Plant-Nematode Interactions; Jones, J., Gheysen, G., Fenoll, C., Eds.; Springer: Dordrecht, Germany, 2011. [CrossRef]

29. Cristóbal, A.J.; Cid del Prado, V.I.; Marbán-Mendoza, N.; Sánchez, G.P.; Mora-Aguilera, G.; Manzanilla, L.R.H. Survival of life stages of Nacobbus aberrans under field conditions. Nematropica 2001, 31, 229-235.

30. Suryawanshi, R.; Chandrashekhar, P.; Hemant, B.; Chandrakant, N.; Lax-mikantSatish, S.P. Nematicidal activity of microbialpigment from Serratia marcescens. Nat. Prod. Res. 2014, 28, 1399-1404. [CrossRef]

31. Marques-Pereira, C.; Proença, D.N.; Morais, P.V. Genome Sequences of Serratia Strains Revealed Common Genes in Both Serratomolides Gene Clusters. Biology 2020, 20, 482. [CrossRef]

32. Wang, S.L.; Lin, C.L.; Liang, T.W.; Liu, K.C.; Kuo, Y.H. Conversion of squid pen by Serratia ureilytica for the production of enzymes and antioxidants. Bioresour. Technol. 2000, 100, 316-323. [CrossRef] [PubMed]

33. Vrain, T.C. A technique for the collection of larvae of Meloidogyne spp. and a comparison of eggs and larvae as inocula. J. Nematol. 1977, 9, 249-251. [CrossRef]

34. Teillet, A.; Dybal, K.; Kerry, B.R.; Miller, A.J.; Curtis, R.H.C.; Hedden, P. Transcriptional changes of the root-knot nematode Meloidogyne incognita in response to Arabidopsis thaliana root signals. PLoS ONE 2013, 8, e61259. [CrossRef]

35. Bankevich, A.; Nurk, S.; Antipov, D.; Gurevich, A.A.; Dvorkin, M.; Kulikov, A.S.; Lesin, V.M.; Nikolenko, S.I.; Pham, S.; Pribelski, A.D.; et al. SPAdes: A new genome assembly algorithm and its applications to single-cell sequencing. J. Comput. Biol. 2012, 19, 455-477. [CrossRef]

36. Wattam, A.R.; Abraham, D.; Dalay, O.; Disz, T.L.; Driscoll, T.; Gabbard, J.L.; Gillespie, J.J.; Gough, R.; Hix, D.; Kenyon, R.; et al. PATRIC, the bacterial bioinformatics database and analysis resource. Nucleic Acids Res. 2014, 42, D581-D591. [CrossRef]

37. Aziz, R.K.; Bartels, D.; Best, A.A.; DeJongh, M.; Disz, T.; Edwards, R.A.; Formsma, K.; Gerdes, S.; Glass, E.M.; Kubal, M.; et al. The RAST Server: Rapid annotations using subsystems technology. BMC Genom. 2008, 9, 75. [CrossRef] [PubMed]

38. Chaumeil, P.A.; Mussig, A.J.; Hugenholtz, P.; Parks, D.H. GTDB-Tk: A toolkit to classify genomes with the Genome Taxonomy Database. Bioinformatics 2019, 36, 1925-1927. [CrossRef]

39. Kejela, T.; Thakkar, V.R.; Thakor, P. Bacillus species (BT42) isolated from Coffea arabica L. rhizosphere antagonizes Colletotrichum gloeosporioides and Fusarium oxysporum and also exhibits multiple plant growth promoting activity. BMC Microbiol. 2016, 18, 277. [CrossRef] 